\title{
Plasmodium Falciparum Malaria and Severe Dengue Coinfection in a Pulmonary Tuberculosis Patient: Case Report and Literature Review
}

\author{
Miño-Bernal Jorge Fernando ${ }^{1 *}$, Martínez Juan Camilo ${ }^{2}$ and Montenegro Ibarra Ángela ${ }^{3}$ \\ ${ }^{1}$ Department of Intensive Care Unit, Clínica las Lajas Ipiales Colombia \\ ${ }^{2}$ Department of Emergency Unit, Clínica las Lajas Ipiales Colombia \\ ${ }^{3}$ Department of Intensive Care Unit, Hospital Civil de Ipiales, Colombia
}

"Corresponding Author: Dr. Miño-Bernal Jorge Fernando, Department of Intensive Care Unit, Clínica las Lajas Ipiales Colombia, Tel: +57 3156737922; E-mail: jorgefernando007@ hotmail.com

Received: 04 April 2018; Accepted: 14 April 2018; Published: 14 May 2018

\begin{abstract}
Malaria and dengue are the most prevalent vector-borne infections in the world. The clinical manifestations of the two pathologies are similar which may complicate the diagnosis of concurrent dengue and malaria. Coinfection causes a clinical picture of greater severity taking into account the proinflammatory state with capillary leakage and alteration of the glucocalix that is triggered. We present the case of an adult patient with coinfection due to malaria and dengue who is also diagnosed with tuberculosis and required management in the intensive care unit due to multiorgan failure.
\end{abstract}

Keywords: Malaria; Plasmodium Falciparum; Dengue; Coinfection

\section{Introduction}

Malaria (caused by parasites of the genus Plasmodium) and Dengue (caused by DENV 1-4 serotypes of dengue virus) are pathologies with a high incidence in tropical countries, and with diverse clinical manifestations that range from mild nonspecific symptomatology to distributive shock and multiple organic failures $[1,2]$. The risk factors that correlate with greater severity are the state of immunosuppression, pregnancy and aging; there are also reports 
of cases that have affirmed the relationship between malaria, chemotherapy, HIV infection and fungal infections [39]; all of which are indispensable factors in generating greater hemodynamic compromise and greater organ damage in the context of a confection of two pathologies transmitted by arboviruses.

The following case report is an example of multiorgan failure related to malaria and dengue confection in a patient with primary pulmonary tuberculosis.

\section{Case Report}

A 36-year-old male patient from Tumaco who consulted for clinical symptoms of 5 days of evolution consisting of subjective thermal increases associated with diffuse abdominal pain, diarrhea without mucus and blood ( 8 times a day) and several episodes of vomiting. He was initially treated at the hospital in Tumaco on 07/05/2017 where they made a thick drop that reported malaria due to Plasmodium falciparum, he was hospitalized for 5 days and discharged home. He goes back to the hospital on 05/12/2017 for exacerbation of the described symptoms. A review by systems: myalgias, polyarthralgias, asthenia, adynamia and hyporexia, signs of respiratory distress so redirect to second level hospital. To the systems review: myalgias, polyarthralgia, asthenia, adynamia and hyporexia, signs of respiratory distress and redirecting to second level hospital.

At physical examination of admission with TA 90/50 mmHg, HR 125 beats per minute, FR 30 breaths per minute. SpO2: $84 \%$ with oxygen supply with Venturi at 30\% (4 liters / minute). Normoreactive isochoric pupils. Cardiac rhythmic noises without murmurs, decreased vesicular murmur in all pulmonary fields with rales of predominance right. Abdomen soft depressible without pain, masses or visceromegalies, delayed capillary filling greater than 3 seconds. Neurological: fluctuation of alertness with Glasgow between 13 and 15.

\subsection{Laboratories}

Positive Thick Drop; IgG and IgM positive for Dengue; smear positive. Blood picture with normocytic normocytic anemia, thrombocytopenia, normal leukocytes with neutrophilia and lymphopenia. Coagulation times slightly prong; impaired liver function tests with hyperbilirubinemia of an obstructive nature; normal kidney function tests. Ionogram with mild hypokalemia and hyponatremia. Arterial gases with moderate decompensated metabolic acidosis with respiratory alkalosis and hyperlactatemia type $\mathrm{A}$, hypoxemia with $\mathrm{Pa} / \mathrm{FiO} 2$ low of 156 . Table 1 shows the values of the initial laboratories.

\begin{tabular}{|l|l|}
\hline \multirow{5}{*}{ Blood count } & Leukocytes: $8.510 \mathrm{millones} / \mathrm{mm}^{3}$ \\
\cline { 2 - 2 } & Neutrophils: $92,1 \%$ \\
\cline { 2 - 2 } & Lymphocytes: $5 \%$ \\
\cline { 2 - 2 } & Hb: $11 \mathrm{gr} / \mathrm{dl}$ \\
\cline { 2 - 2 } & Hto: $33 \%$ \\
\cline { 2 - 2 } & Platelets: $64000 / \mathrm{mm}^{3}$ \\
\hline
\end{tabular}




\begin{tabular}{|c|c|}
\hline \multirow{3}{*}{ Coagulation times } & TP: $15,7 / 14 \mathrm{seg}$ \\
\hline & TPT:40/28 seg \\
\hline & INR: 1,16 \\
\hline \multirow{2}{*}{ Renal function } & Creatinine: $1,2 \mathrm{mg} / \mathrm{dl}$ \\
\hline & BUN:39,2 mg/dl \\
\hline \multirow{4}{*}{ Liver function } & AST:36 U/L \\
\hline & ALT:29 U/L \\
\hline & Total bilirubin: $4,22 \mathrm{mg} / \mathrm{dl}$ \\
\hline & Direct bilirubin: $2,56 \mathrm{mg} / \mathrm{dl}$ \\
\hline Glucose & $85 \mathrm{mg} / \mathrm{dl}$ \\
\hline \multirow{3}{*}{ Electrolytes } & Sodium $132 \mathrm{mEq} / \mathrm{L}$ \\
\hline & Chlorine $105 \mathrm{mEq} / \mathrm{L}$ \\
\hline & Potassium 3,1 mEq/L \\
\hline \multirow{8}{*}{ Arterial gases } & $\mathrm{pH}: 7.44$ \\
\hline & PCO2: $24 \mathrm{mmHg}$ \\
\hline & PO2: $47 \mathrm{mmHg}$ \\
\hline & HCO3: $16.3 \mathrm{mEq}$ \\
\hline & EB: -7.9 \\
\hline & $\mathrm{Pa} / \mathrm{FiO} 2: 156$ \\
\hline & SO2:88\% \\
\hline & Lactate: $4,3 \mathrm{mmol} / \mathrm{L}$ \\
\hline
\end{tabular}

Table 1: The values of the initial laboratories.

\subsection{Electrocardiogram}

Sinus tachycardia with HR of 110 beats per minute, PR $0.16 \mathrm{sec}$, QRS of normal morphology with duration of 0.12 sec, ST segment and T wave without alterations.

\subsection{Chest $\mathrm{x}$-rays that report}

Presence of alveolar opacities that compromise both lung fields in 3 of 4 quadrants with bilateral obliteration of cost phrenic angles, pulmonary hilies and vascular pattern are preserved, cardiopericadic silhouette and mediastinal structures of normal characteristics. 


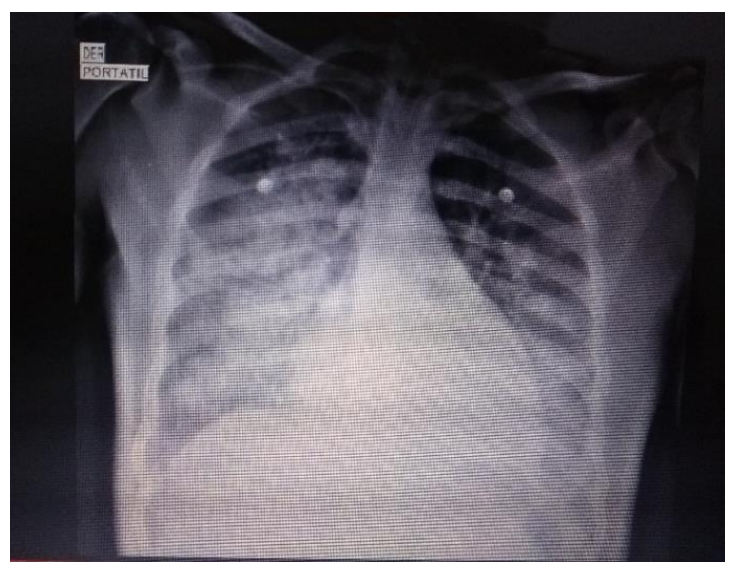

Figure 1: Chest x-ray. Anteroposterior projection.

Simple brain tomography reported as normal. A high resolution chest CT scan is performed showing right lung cavitation, bronchopneumonia, bilateral basal segmental atelectasis and right pleural effusion (Figures 2 and 3). Total abdomen ultrasound showing hepatosplenomegaly.

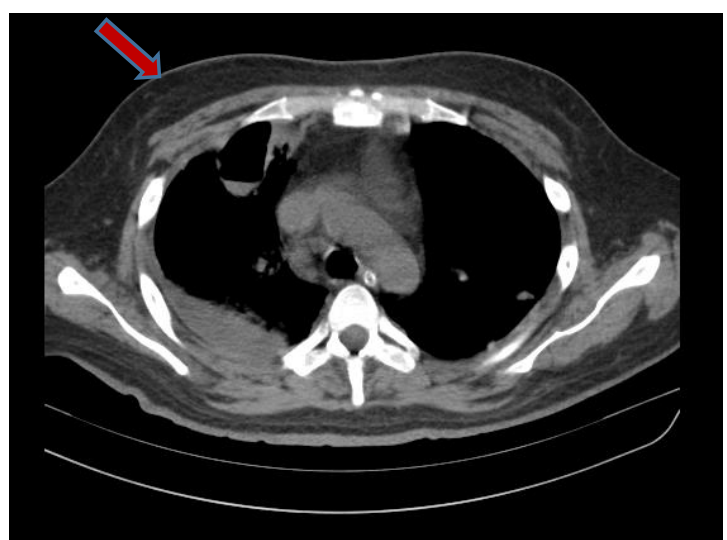

Figure 2: Chest tomography: the arrow indicates the presence of a pulmonary cavitation.

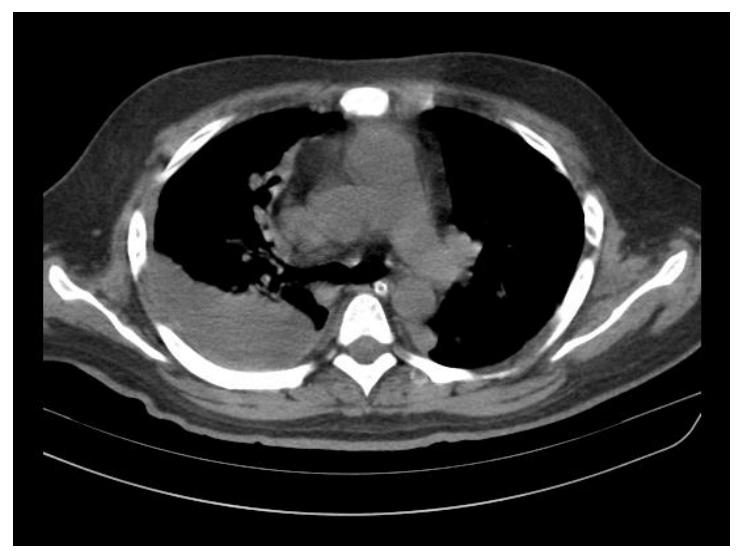

Figure 3: Chest tomography showing right pleural effusion. 
The patient is diagnosed with falciparum plasmodium malaria in resolution, hemorrhagic dengue, pulmonary tuberculosis, adult respiratory distress syndrome, metabolic acidosis and hydroelectrolytic disorder. The patient is hemodynamically stabilized with balanced solutions, he also requires invasive mechanical ventilation with protective parameters, norepinephrine management and prophylaxis of stress ulcers as well as initiation of tetraconjugated antituberculous therapy, and antimalarial arthemeter and lumefantrine (Coartem). Considering his conditions he moves to the intensive care unit for handling.

\section{Discussion}

Malaria and dengue are typical diseases of residents and travelers in tropical countries [10]. In relation to malaria is a disease caused by the parasite of the genus Plasmodium, transmitted by the bite of the mosquito Anopheles. In humans it grows and multiplies in hepatocytes and erythrocytes. When gametocytes are taken by the female Anopheles, the sexual reproduction of the parasite begins in its intestine resulting in a zygotic form, which leads to the formation of oocysts and sporocysts. After 10-18 days sporozoites are found in the salivary glands of the mosquito infecting a new individual and repeating cycle [11].

In 2010, there were 1,133,000 deaths worldwide due to malaria, with Africa being the continent where most cases are reported, 70 million in 2009. The majority of reported cases are related to Plasmodium falciparum infection, with Haiti, Suriname and Guyana being the countries with the highest prevalence [12].

Dengue is part of the family of Flaviridae virus (Serotypes DEN 1, 2, 3, 4), is transmitted by the Aedes aegpti mosquito with a human-vector-human transmission cycle, the virus replicates in the intestine and in the salivary glands of the mosquito transmitting to other individuals [11]. Dengue infection is endemic in more than 100 countries, increasing its incidence 30 times in the last 50 years. In 2013, it was estimated that more than 390 million people worldwide were infected [2].

These pathologies may manifest severely with target organ injury. In the case of infection by Plasmodium falciparum, this generates the expression of erythrocyte membrane protein of P. falciparum type 1 (Pfemp1), which binds the erythrocyte to the endothelial wall, specifically to the endothelial receptor of protein C (EPCR), generating capillary leakage as well as additional release of proinfalmatory cytokines type TNFalpha, and IL1, and increased expression of ICAM-1 [13]. On the other hand, severe dengue coinfection is also implicated in hypovolemic shock with similar molecular mechanisms with overexpression of TNFalpha, IL6, IL8, IL10, IL12, macrophage migration inhibitory factor, NS1 protein and extracellular matrix metalloproteinases. Finally, depletion of the endothelial glycocalyx has been proposed as a factor that reduces intravascular oncotic pressure by perpetuating the mentioned lesions [2].

Plasmodium falciparum infection is most commonly associated with severe malaria and increased mortality due to elevated levels of IL-1B and the sequestration of mature forms in microvascular beds, which contributes to 
metabolic acidosis, hyperlactatemia and white-body involvement [14]. The patient entered the intensive care unit with criteria of severe malaria given by vasodilated (septic) and hypovolemic shock for the previously described conditions, in addition to Berlin criteria for moderate ARDS (4 quadrant infiltrates, $\mathrm{Pa} / \mathrm{FiO}_{2}=112$ ) [15-19], coagulopathy (INR Prolonged, thrombocytopenia) [20], hemodynamic instability, shock Liver (elevated bilirubin) [21] and neurological compromise due to fluctuation of alertness [21]. In other patients, acute renal injury may also occurs [23].

In addition to those mentioned, he also presents with normocytic normocytic anemia and metabolic acidosis, which are secondary to the proinflammatory effect, hemolysis (less deformity, dyseritropoiesis), endothelial reticulum clearance, and anaerobic glycolysis $[24,12]$. The semiological finding of splenomegaly is evident in $40 \%$ of the cases, being more frequent, in some series, in serious presentations [25].

The risk factors to consider greater severity in malaria infection are; [26, 27]

- Species of Plasmodium falciparum

- Children between 6 months and 5 years

- Asian, Caucasian

- Sickle cell disease

- Prior exposure to antimalarial drugs and therapeutic failure

- Therapeutic delay

- Tourist condition

The first reported case of Malaria and dengue co-infection in the world occurred in France in 2005, after which there is a report of 36 cases in Asia, which are manifested by severe clinical conditions [11]. This particular case also presents Dengue coinfection, demonstrated by positive IgG and IgM levels, thus increasing morbidity through increased hemodynamic compromise; Some reports such as that of Assir et al. Consider that this association is also correlated with jaundice as a parameter of severity [28]. The most frequent laboratory values in malaria and dengue coinfections are thrombocytopenia, anemia and elevation of transaminases AST < ALT.

Malaria plus Dengue co-infection is associated with other pathogens such as Hepatitis A, Hepatitis E, Chikungunya, Leptospirosis, Filariasis, Typhoid fever, Leptospirosis [11]. However, there is no case report of this coinfection plus tuberculosis being this patient the first case worldwide. Animal models that have studied Malaria-Tuberculosis coinfection have found associations with ARDS and cerebral malaria, further shading the clinical prognosis of affected patients [29].

\section{Why Should an Emergency Physician Take Account of This Disease?}

Malaria is a common pathology in tropical countries that can manifest itself severely with multiple organ failure, especially in the presence of comorbidities that condition a probable state of immunosuppression such as 
Tuberculosis or diseases that enhance the state of shock such as Dengue. The emergency physician should bear in mind the presence of coinfections which may occur in sudden-onset unexplained febrile spikes in patients with baseline Tuberculosis, multiple organ failure with unpredictable clinical courses and haemorrhagic complications which are highly likely to occur in cases of severe Dengue [30].

\section{Competing Interests}

The authors declare that they have no competing interests.

\section{References}

1. Janneck L, Koyfman A, Takayesu JK. Clinical review of malaria for the emergency physician Evaluation clinique du paludisme pour les medecins urgentistes African Journal of Emergency Medicine 1 (2011): 126-130.

2. Guzman MG, Harris E. Dengue. Lancet 385 (2015): 453-465.

3. Walker NF, Nadjm B, Whitty CJM. Malaria. Medicine 42 (2014): 100-106.

4. Focà E, Zulli R, Buelli F, et al. Recrudescenza di malaria da P. falciparum in un paziente neoplastico Le Infezioni in Medicina 1 (2009): 33-34.

5. Imani PD, Musoke P, Byarugaba J, et al. Human immunodeficiency virus infection and cerebral malaria in children in Uganda: a case-control study BMC Pediatrics 11 (2011): 5.

6. Laufer MK, van Oosterhout JJG, Thesing PC, et al. Malaria Treatment Efficacy among People Living with HIV: The Role of Host and Parasite Factors. Am. J. Trop. Med. Hyg 77 (2007): 627-632.

7. Slutskera L, Marston BJ. HIV and Malaria: interactions and implications, Current Opinion in Infectious Diseases 20 (2007): 3-10.

8. Skinner-Adams TS, McCarthy JS, Gardiner DL. HIV and malaria co-infection: interactions and consequences of chemotherapy Trends in Parasitology 24 (2008): 264-271.

9. Däbritz J, Schneider M, Just-Nuebling G, et al. Minireview: invasive fungal infection complicating acute Plasmodium falciparum malaria Mycoses 54 (2009): 311-317.

10. Taylor SM, Molyneux ME, Simel DL, et al. Does This Patient Have Malaria?. JAMA 304 (2010): 20482056.

11. Selvaretnam AP, Sahu PS, Sahu M. A review of concurrent infections of malaria and dengue in Asia Asian Pac J Trop Biomed 6 (2016): 633-638.

12. Cheng MP, Yansouni CP. Management of Severe Malaria in the Intensive Care Unit. Crit Care Clin 29 (2013): 865-885.

13. Marks M, Gupta-Wright A, Doherty JF, et al. Managing malaria in the intensive care unit British Journal of Anaesthesia 113 (2014): 910-921.

14. Cowman AF, Healer J, Marapana D. Malaria: Biology and Disease Cell 167 (2016): 610-624.

15. Committee and the Members of the ARDS Definition Task Force Acute Respiratory Distress Syndrome. The Berlín Definition JAMA 307 (2012): 2526-233. 
16. Habib AG, Singh KS. Respiratory Distress in Nonimmune Adults with Imported Malaria. Infection 32 (2004): 356-359.

17. Lomar AV, Vidal JE, Lomar FP, et al. Acute Respiratory Distress Syndrome Due to Vivax Malaria: Case Report and Literature Review. The Brazilian Journal of Infectious Diseases 9 (2005): 425-430.

18. Cabezón Estévanez I, Hernández-Mora MG. Pulmonary complications of malaria: An update. Med Clin (Barc) 146 (2016): 354-358.

19. Van den Steen PE, Deroost K, Deckers J. Pathogenesis of malaria-associated acute respiratory distress syndrome Trends in Parasitology 29 (2013): 346-358.

20. Angchaisuksiri P. Coagulopathy in malaria Thrombosis Research 133 (2014): 5-9.

21. Frevert U, Nacer A, Cabrera M, et al Imaging Plasmodium immunobiology in the liver, brain, and lung. Parasitol Int 63 (2014): 171-186.

22. Hora R, Kapoor P, Thind KK. Cerebral malaria - clinical manifestations and pathogenesis Metab Brain Dis 31 (2016): 225-237.

23. Chellappan A, Bhadauria DS. Acute kidney injury in malaria: An update Clinical Queries: Nephrology 5 (2016): 26-32.

24. Nadjm B, Behrens RH. Malaria: An Update for Physicians, Infect Dis Clin N Am 26 (2012): 243-259.

25. Nilles EJ, Arguin PM. Imported malaria: an update. American Journal of Emergency Medicine 30 (2012): 972-980.

26. Basu S, Sahi PK. Malaria: An Update. Indian J Pediatr 30 (2017): 1-8.

27. Lüthi B, Schlagenhauf P. Risk factors associated with malaria deaths in travellers: A literature review. Travel Medicine and Infectious Disease 13 (2015): 48-60.

28. Assir MZK, Masood MA, Ahmad HI. Concurrent dengue and malaria infection in Lahore, Pakistan during the 2012 dengue outbreak. International Journal of Infectious Diseases 18 (2014): 41-46.

29. Mueller AK, Behrends J, Blank J, et al. An Experimental Model to Study Tuberculosis-Malaria Coinfection upon Natural Transmission of Mycobacterium tuberculosis and Plasmodium berghei. J.Vis.Exp 84 (2014): 1- 8 .

30. Joob B, Wiwanitkit V. Concurrent dengue infection in a patient with pulmonary tuberculosis: the first world report Thorac Dis 5 (2013): E118-E119.

Citation: Miño-Bernal Jorge Fernando, Martínez Juan Camilo, Montenegro Ibarra Ángela. Plasmodium Falciparum Malaria and Severe Dengue Coinfection in a Pulmonary Tuberculosis Patient: Case Report and Literature Review. Archives of Clinical and Medical Case Reports 2 (2018): 82-89. 